\title{
Screening for comorbidities in COPD
}

Due to growing recognition of comorbidities, COPD is no longer considered a disease affecting only the respiratory system. Its management now entails the early diagnosis and treatment of comorbidities. However, although many studies have examined the impact of comorbidities on the evolution of COPD and patients' quality of life, very few have explored the means to systematically identify and manage them. The aims of this article are to summarise the state of current knowledge about comorbidities associated with COPD and to propose a possible screening protocol in the outpatient setting, emphasising the areas needing further research.
Traditionally, severity of COPD has been assessed using spirometry, quantifying airflow limitation in terms of forced expiratory volume in $1 \mathrm{~s}\left(\mathrm{FEV}_{1}\right)$. However, there is a weak correlation between $\mathrm{FEV}_{1}$, symptoms and global health status. Thus, in 2011, the Global Initiative for Chronic Obstructive Lung Disease (GOLD) strategy redefined COPD as a multisystem disease, whose assessment should incorporate measurement of symptoms and $\mathrm{FEV}_{1}$, history of exacerbations, and identification and management of comorbid diseases [1]. A new chapter appeared in the GOLD strategy document at that time, renewed each year, highlighting the importance of comorbidities in COPD, especially cardiovascular disease, anxiety, depression, osteoporosis and lung cancer.

Now, 9 years later, despite abundant research on the prevalence of comorbid diseases and the interactions between these diseases and COPD, we are still lacking exact recommendations about which comorbidities to screen for, how often and the best means of screening. For many comorbid diseases, the impact of treatment on the natural history of COPD is also unclear. In fact, looking into screening for comorbidities in COPD raises more questions than answers, but is a unique opportunity to explore new perspectives.

This review will first revise the definitions of comorbidity and screening. Secondly, we will put forward a list of the COPD comorbidities most relevant for screening. Thirdly, we will describe current knowledge and screening tools for each comorbidity. Fourthly, we propose a simple, structured checklist of comorbidities in COPD, which can be used in outpatient consultation.

\section{Definitions}

\section{Comorbidity}

There is no absolute definition of comorbidity. It can be simply defined as one or more diseases coexisting with another primary disease of interest
Cite as: Natali D, Cloatre G, Hovette P, et al. Screening for comorbidities in COPD. Breathe 2020; 16: 190176. 
(index disease) [2]. Diseases may coexist within the same individual by chance or by causal association. In turn, the causal association may be explained by a shared risk factor or comparable underlying pathological processes. Comorbidity raises other issues, including disease interactions and increases in symptom burden and patient complexity.

\section{Screening}

According to the World Health Organization, screening is "the presumptive identification of unrecognized disease in an apparently healthy, asymptomatic population by means of tests, examinations or other procedures that can be applied rapidly and easily to the target population" [3]. Screening is therefore a process with three crucial components: 1 ) the ability to predict that a screened individual has the disease; 2) the capability to correctly establish a diagnosis; and 3) the means to treat the detected disease. Thus, screening is relevant if there is an accurate diagnostic test and an effective treatment that provides improvement in the course, symptoms and/or mortality of the disease. Both the diagnostic test and the treatment offered should have acceptable harm and cost when weighed against the expected benefits.

\section{Which comorbidities should we screen in COPD?}

Increasingly, COPD is being recognised as a multisystem disease. Some associated diseases have obvious consequences for COPD and are easily detectable in daily practice. For example, chronic dental disease or sinus infection, deglutition disorders, bronchiectasis, allergic disease and acquired or congenital immune deficiency disorders may increase the risk for exacerbation. However, for other comorbid diseases, the relationship is more obscure. Several large database studies, summarised in review articles by CHATILA et al. [4] and Acusti and Soriano [5], indicate an excess of cardiovascular diseases (coronary artery disease, arrhythmia, cardiac failure), metabolic diseases (diabetes mellitus, dyslipidaemia, obesity, osteoporosis, sarcopenia), psychological disorders (anxiety, depression), lung cancer, sleep disruption and sleep disordered breathing, and gastro-oesophageal reflux disease (GORD) in COPD. Each comorbidity adds to the complexity of COPD management, affecting the rate of hospitalisations and exacerbations, the duration of inpatient stay, the cost of medical care and mortality. Furthermore, some of these comorbidities are particularly strong predictors of mortality. Divo et al. [6] quantified the prevalence and mortality risk of comorbidities in a prospective observational cohort study, finding that coexisting anxiety, malignancy or cardiac disease was strongly associated with adverse prognosis.
Table 1 Proposed list of conditions that warrant screening in COPD

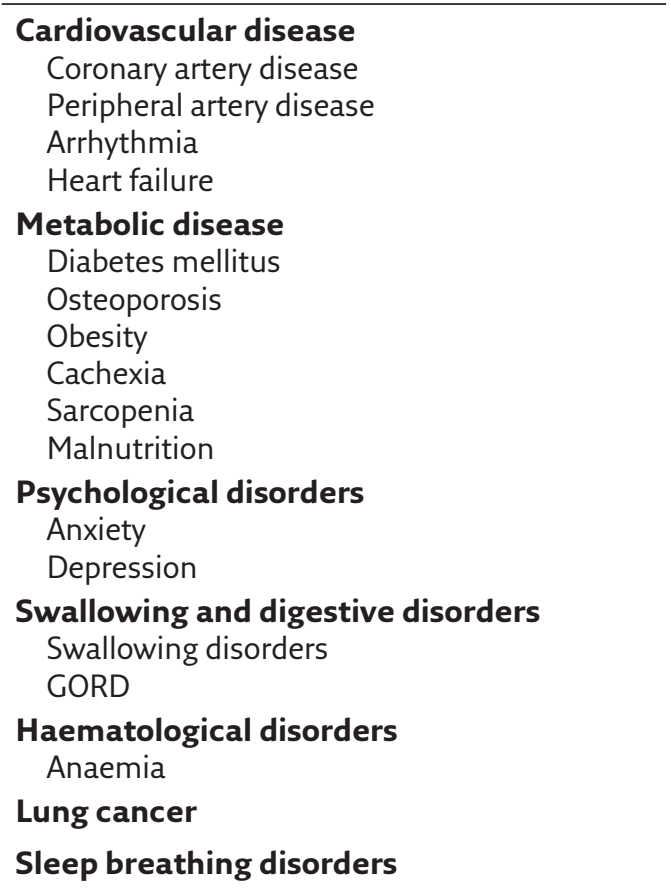

Table 1 illustrates our proposed list of conditions that warrant screening in COPD. However, the screening process should be further individualised, according to the patients' predominant symptoms and phenotypic manifestations.

\section{Why is it relevant to screen for comorbidity during a chronic illness?}

Various comorbidities may change the evolution of COPD, and may affect the rate of deterioration, symptom burden, frequency or severity of exacerbations, threshold for hospitalisation and mortality. There may be implications for treatment, including drug or disease interactions and compliance.

\section{Mechanisms for interactions between COPD and its associated comorbidities}

The association between COPD and some comorbidities may be partly due to shared risk factors (e.g. age, tobacco smoke, sedentary lifestyle). However, there is increasing evidence of overlapping pathophysiology, including low-grade systemic inflammation, which contributes to coronary artery disease, osteoporosis, sarcopenia and possibly depression [7]. Another example is autonomic 
dysfunction with excess sympathetic nervous system activity, which is recognised in $\operatorname{COPD}[8,9]$ but may also be implicated in anxiety, cardiac failure or dysrhythmia.

Treatment of comorbidities may influence symptoms, progression or complications of COPD per se or, alternatively, treatment may target shared disease mechanisms. Probably the most obvious impact on management is to increase the number of medications and the complexity of the treatment regimen, with increased potential for drug interactions, side-effects, prescribing errors and lapses in adherence. Polypharmacy is certainly a well-recognised issue: in the UK Biobank database [10], 52\% of 8317 self-reported COPD patients had more than five medications and $15.3 \%$ had more than 10 medications, compared with $17.7 \%$ and $2.2 \%$ respectively in 494323 control patients $(p<0.001)$. COPD patients taking more than three medications were more likely to have sideeffects, such as falls, constipation, urine retention, depression, bleeding or renal injury.

\section{How to screen for comorbidities in COPD}

Screening is feasible if both a reliable diagnostic test and an effective treatment are available. In addition, the combined risks and expense of testing and treatment should be weighed against the potential benefit of treatment before proceeding. However, although screening is feasible for several comorbid diseases in COPD, there is no consensus or guideline about an appropriate screening approach. Moreover, COPD may complicate identification and diagnosis of comorbidities: symptoms of COPD and comorbid conditions may overlap, leaving the second condition unrecognised, or the presence of coexistent COPD may present challenges to diagnostic testing.

\section{Clinical evaluation}

Clinical evaluation and building a therapeutic relationship initially require the establishment of rapport, respect and trust between the patient and the clinical team. The first step is to obtain a detailed medical history and physical examination, targeting those comorbidities that seem most relevant to symptom burden, prognosis and quality of life (table 1). Importantly, the evaluation should be updated at each encounter, seeking evidence of new diagnoses, treatments and side-effects. Unfortunately, clinical evaluation alone is usually not sufficiently sensitive to confirm the existence of comorbidity. However, it is useful to direct further diagnostic testing.

The following sections concentrate on comorbid conditions that may be difficult to identify or where the diagnostic approach differs in the setting of
COPD. Potential treatments with therapeutic benefit are also discussed. Diseases that may be easily identified and treated by conventional means are not further discussed here.

\section{Cardiovascular disease}

"Cardiovascular disease" is a nonspecific term that commonly includes coronary artery disease, cerebrovascular disease, peripheral artery disease, arrhythmia and heart failure. It is more frequent in patients with COPD than in the population without COPD and, when present, is associated with worse prognosis. Coexistence may be due to shared risk factors, such as cigarette smoking, ageing, sedentary behaviour and low socioeconomic status. However, reduced $\mathrm{FEV}_{1}$ per se is an independent risk factor for cardiovascular death, on a par with dyslipidaemia and hypertension. In COPD, a 10\% reduction in $\mathrm{FEV}_{1}$ is accompanied by a $28 \%$ increase in cardiovascular mortality [11, 12]. Pathological mechanisms common to both diseases, such as chronic systemic inflammation, oxidative stress and sympathetic nervous system overactivity, are certain to be key factors contributing to the relationship between COPD and cardiovascular disease.

Coronary artery disease worsens COPD outcomes, with increased exacerbations, hospitalisations, mortality and health costs. Cardioselective beta-blockers [13, 14], hydroxymethylglutarate-CoA reductase inhibitors (statins) and antiplatelet agents confer benefit for coronary artery disease and are safe to use in COPD patients. Likewise, angiotensin-converting enzyme inhibitors and beta- 1 selective blockers are recommended treatments in cardiac failure, regardless of whether the patient has COPD. Longacting bronchodilators are generally safe in COPD patients with coexistent coronary artery disease, provided that they are used at the recommended dose and frequency. Revascularisation procedures may be beneficial but are associated with increased risk of mortality in COPD $[15,16]$. Percutaneous procedures confer less risk [16] but sometimes present technical challenges, due to the patients' inability to lie flat.

Reduced $\mathrm{FEV}_{1}$ is a risk factor for atrial fibrillation [17], which is a frequent complication during COPD exacerbation. Data from both retrospective [18] and prospective [19] cohort studies suggest that the presence of atrial fibrillation worsens the risk of mortality in COPD, particularly in the setting of respiratory exacerbation [18]. Arrhythmogenicity may be increased in COPD patients because of hypoxaemia, hypercapnia, pulmonary hypertension and ventricular diastolic dysfunction, oxidative stress and inflammation, and the side-effects of $\beta$-agonists, anticholinergics, systemic corticosteroids and theophylline. Antiarrhythmic drugs such as beta-blockers, accessory pathway ablation and ventricular pacing are potential treatment options. 
Diagnostic difficulties

Diagnostic difficulties that may be encountered while screening for cardiovascular disease include the following: 1) ischaemic heart disease may present without typical anginal symptoms in patients with COPD; 2) dyspnoea, diminished exercise capacity or chest tightness caused by coronary artery disease or cardiac dysfunction are difficult to distinguish from those caused by COPD; and 3) arrhythmia may be intermittent and/or asymptomatic and so may remain unnoticed until a subsequent cerebrovascular event.

Potential screening tools

CAzzola et al. [20] proposed a simple and costless algorithm to assess the risk of coronary artery disease in COPD patients and to help select patients for further investigations, termed "COPDCoRi", based on sex, dyslipidaemia and smoking habits. This algorithm was built using retrospective data from COPD and non-COPD patients undergoing a coronary arteriography in a single institution.

Noninvasive diagnostic tools can be difficult to interpret in COPD patients. A resting ECG may show signs of ischaemic events or diagnose cardiac arrhythmia. An abnormal exercise stress test, using an ECG, may prove diagnostic for coronary artery disease, although limitations include exercise intolerance due to lung function impairment or peripheral artery disease. Alternatively, pharmacological stress testing or radionuclide studies may be undertaken. Markers of myocardial damage, such as cardiac troponin, may be elevated without detectable coronary disease and markers of cardiac stretch, such as brain natriuretic peptide (BNP), may be chronically elevated and reflect either left- or right-sided cardiac dysfunction. However, normal values, particularly for BNP, are reassuring in terms of prognosis. Echocardiography may provide valuable diagnostic information about cardiac chamber size, valvular heart disease, ventricular function and pulmonary hypertension. However, the interpretation may be limited by poor echogenicity due to severe emphysema. Computed tomography coronary angiography (CTCA) with calcium scoring is a newer approach to diagnosing coronary artery disease in COPD patients, as demonstrated in a prospective observational cross-sectional study by OzYILMAz et al. [21] comparing 42 COPD GOLD stage II patients and 31 healthy control subjects. However, interpretation may be limited by tachycardia. Moreover, inability to tolerate supine posture may present practical issues in performing CTCA or conventional coronary angiography. Cardiac magnetic resonance imaging may prove an interesting tool, enabling reliable evaluation of left ventricular function, right chambers and pulmonary arteries, although this too has clinical limitations related to supine posture, claustrophobia and tachycardia, as well as healthcare system issues related to availability and expense.

\section{Metabolic diseases}

Diabetes mellitus

Most commonly, COPD is associated with type Il diabetes mellitus rather than type I disease. Diabetes combined with COPD worsens prognosis, being associated with higher susceptibility to respiratory infections (including pneumonia), prolonged hospital stay for COPD exacerbations and increased mortality [22]. The mechanism of interaction between these two diseases and the resultant adverse effects on disease outcomes are not clearly understood, although smoking, effects of sustained hyperglycaemia on lung physiology, systemic inflammation, susceptibility to infections, and treatment effects of systemic corticosteroids may well all be implicated. Screening strategies for diabetes in COPD patients do not differ from those used in patients without COPD. However, it is interesting to note that two recent retrospective cohort studies, one smaller (4231 COPD patients [23]) and one larger (19505 COPD patients with diabetes using metformin, compared with 19505 COPD patient controls [24]), have suggested increased survival in COPD patients with diabetes mellitus and metformin treatment, paving the way for further prospective studies of this drug, investigating outcomes related to diabetes, cardiovascular disease and COPD.

\section{Osteoporosis}

The increased prevalence of osteoporosis seen in COPD is attributed to systemic inflammation, smoking, physical inactivity, low body mass index (BMI), malnutrition, hypogonadism and use of systemic corticosteroids. Osteoporosis-related vertebral fractures may adversely affect lung function and, as with other fractures, may impair mobility or worsen quality of life. Pulmonary rehabilitation or other forms of weight-bearing physical activity may improve osteoporosis and osteopenia in COPD. Supplementation with calcium and vitamin $D$ should be considered in those with dietary insufficiency, and antiresorptive therapies are recommended for those with established osteoporotic fractures or deteriorating bone mineral density measures in the setting of ongoing risks for osteoporosis.

Potential screening tools for osteoporosis include a pragmatic, five-step approach developed by Romme et al. [25] to screen COPD patients at high risk of osteoporosis. This approach incorporates a risk stratification tool, diagnostic testing with dual-energy X-ray absorptiometry (DXA) of the 
spine, assessment for potential secondary causes, a multifaceted lifestyle intervention directed towards osteoporosis prevention, and ongoing monitoring of compliance, efficacy and side-effects.

Other disorders of metabolism: obesity, malnutrition, cachexia and sarcopenia

Being overweight or underweight are both conditions prevalent in COPD patients, with important implications for morbidity and mortality. $\mathrm{BMI}$ has an interesting relationship with mortality in COPD patients. Being underweight increases mortality risk. Being overweight is protective, to a point. However, once BMl exceeds $32 \mathrm{~kg} \cdot \mathrm{m}^{-2}$, this survival benefit is lost [26].

The most consistent lung function abnormality in obese patients with COPD, in addition to the diagnostic criteria of fixed airflow limitation on spirometry, is a reduced expiratory reserve volume. This may contribute to the worse dyspnoea, increased reliever medication use, reduced healthrelated quality of life and decreased exercise capacity generally seen in obese patients with COPD. Sedentary behaviour, smoking cessation and systemic corticosteroid use are factors in COPD patients that may increase the risk of obesity.

Malnutrition may be overlooked in obese COPD patients but should be carefully considered in these patients as well as those COPD patients who are underweight or cachectic, as malnutrition predicts increased healthcare utilisation and mortality [27].

The reasons behind cachexia in COPD patients may include reduced energy intake (related to anorexia, fatigue or the impact of breathlessness on swallowing ability) or increased energy consumption (with increased respiratory muscle work and catabolism from systemic inflammation and oxidative stress), resulting in negative energy balance, sarcopenia and muscle weakness. Importantly, introducing nutritional support has been demonstrated to be effective for increasing muscle strength and exercise tolerance in cachectic COPD patients [28]. Benefits are more certain if supplementation begins pre-emptively, before malnutrition is well established [29].

Sarcopenia affects exercise performance and quality of life. Systemic inflammation, oxidative stress, inactivity (deconditioning), use of systemic corticosteroids or malnutrition may be contributing factors. Pulmonary rehabilitation is an effective treatment for sarcopenia, especially if contributing factors can also be addressed [30].

Diagnostic difficulties may occur, as reliable and accurate assessment of nutrition in COPD is complex. A screening test such as measurement of BMI can measure obesity, but does not reliably detect malnutrition or sarcopenia. The European Working Group on Sarcopenia in Older People has produced a set of diagnostic criteria for sarcopenia, depending on muscle mass and muscle function (strength or performance) [31]. Muscle mass is assessed by bioelectrical impedance analysis, DXA and anthropometry. Muscle strength is measured by handgrip strength; physical performance is evaluated by usual gait speed and a short physical performance battery. Body composition estimates of muscle and fat components do provide additional information but may not be readily accessible in the clinic setting. Serum albumin level is often used as a readily available marker of malnutrition, although the level is usually reduced in the setting of inflammation. Dietitian assessment combined with other routine haematology and biochemistry testing, including haematinics and selected vitamin and mineral levels, may provide an approximate gauge of nutritional status in the absence of more sophisticated testing methods.

\section{Psychological disorders}

Depression and anxiety are common in COPD patients, and are associated with poor quality of life, reduced adherence to treatment, higher rates of re-admission after acute exacerbation and increased mortality [32, 33]. Dyspnoea, $\beta$-agonist medication, chronic hypoxaemia, reduced physical activity, lack of recreational activity, poor sleep and solitude are potential contributing factors. Some medications used in anxiety and depression may have respiratory depressant or sedative effects, with implications for COPD patients with respiratory failure. However, pulmonary rehabilitation has been shown to relieve dyspnoea and improve anxiety and depression [34].

\section{Diagnostic difficulties}

Psychological disorders may be challenging to diagnose in COPD patients, because symptoms of anxiety and depression may be reactive and appropriate to individual patient circumstances, particularly if patients are physically deteriorating, becoming less independent and more reliant on assistance from others for routine daily tasks. Moreover, anxiety per se can increase dyspnoea and induce tachypnoea, contributing to hyperinflation and gas trapping, worsening COPD symptoms.

\section{Potential screening tools}

Different scoring systems are validated to screen anxiety and depression in COPD: the Hospital Anxiety and Depression scale (HADS) score is widely used, the 25-item Hopkins Symptom Checklist (HSCL-25) and Clinical COPD Questionnaire (CCQ) scores help to distinguish those patients in need for psychological care, and the Beck Depression Inventory (BDI) score may identify depression in COPD patients. However, these tools are a preliminary means of assessing depression and anxiety, in order to select those patients who may 
need further assessment. They in no way replace mental state examination and detailed psychological assessment, which should be undertaken by a clinician with the appropriate expertise.

\section{Disorders of swallowing and digestion}

Swallowing and breathing are coordinated processes, requiring timely closure of the larynx to facilitate transit of ingested food material appropriately along the oesophagus towards the stomach, and preventing inappropriate passage of foodstuffs beyond the larynx into the lower respiratory tract. COPD patients may have a lack of synchronisation between breathing and swallowing, particularly in the setting of symptomatic worsening or exacerbation, leading to an increased risk of aspiration pneumonitis and a subsequent tendency to higher rates of exacerbation. Mucosal dryness from antimuscarinic bronchodilator medications may also contribute to swallowing difficulties.

GORD is a common disorder, both in the general population and in COPD. In COPD patients, prevalence of symptomatic GORD is increased in comparison with that seen in the normal population, and its presence is predictive for COPD exacerbations [35]. It may be due to altered pressures in the lower oesophageal sphincter and to side-effects of respiratory medications. Lifestyle modifications are suggested, including avoiding supine posture after eating and dietary adjustment (avoiding foods known to precipitate reflux symptoms, reducing meal quantity and earlier scheduling of the meal prior to bedtime). Pharmacological acid suppression therapy with proton pump inhibitors or histamine- 2 receptor blockers is effective in reducing symptoms of GORD but does not prevent reflux of gastric contents. The impact of pharmacological treatment for GORD on exacerbations in COPD patients is controversial.

\section{Diagnostic difficulty}

Disorders of swallowing and GORD may be asymptomatic. The symptoms of either (breathlessness, wheeze and cough) may be mistaken for symptoms of COPD and either may present as COPD exacerbation.

\section{Potential screening tools}

There are several screening tools for oropharyngeal dysphagia, which may be used in the setting of increased aspiration risk. These take the form of questionnaires (such as Eating Assessment Tool10 (EAT-10) [36]) and clinician-administered "bedside" swallowing assessments requiring a variable degree of clinician expertise. A recent prospective study used a 9 -item questionnaire, a water swallow test and a cookie swallow test to screen for dysphagia in 51 stable COPD outpatients [37]. These are simple, costless tools, which can be easily used in daily practice. They detected $65 \%$ of patients with subjective signs of swallowing disorders and $49 \%$ with objective dysfunction on swallowing tests. However, probably the most readily available and sensitive test is submandibular videofluoroscopy, which may be overseen by a speech therapist and objectifies laryngeal penetration and aspiration [38]. Typical GORD symptoms are temporally associated with food intake and supine posture. Ambulatory 24-h oesophageal $\mathrm{pH}$ monitoring is the gold standard to diagnose GORD but may be unnecessary if a trial of lifestyle measures and pharmacotherapy results in relief of symptoms.

\section{Haematological disorders}

Polycythaemia is traditionally associated with chronic hypoxia. Therefore, low or inappropriately normal haemoglobin levels in this group may contribute to breathlessness and/or represent dietary insufficiency or chronic, occult blood loss. Anaemia may also be due to systemic inflammation, dysregulation of the renin-angiotensin-aldosterone system or renal impairment. Anaemia due to iron deficiency can be successfully treated with intravenous supplementation. Oral supplementation is also effective but depends on tolerance of side-effects, such as constipation, and the ability to absorb iron via the gastrointestinal tract. Erythropoietin supplementation may be offered for patients with coexisting renal dysfunction. Ideally, the underlying cause of anaemia should be also addressed, if possible.

Diagnostic difficulties may be encountered because COPD and anaemia have overlapping symptoms (asthenia, dyspnoea, exercise intolerance). Iron status, ferritin, transferrin saturation and total binding capacity measurements are altered in inflammation and may lead to underdiagnosis of iron deficiency in COPD. Testing for anaemia with full blood examination and iron studies, including serum iron, ferritin levels and transferrin saturation are inexpensive tests and are recommended to screen patients with disproportionate breathlessness or with risk factors for anaemia on clinical assessment. Iron studies may be interpreted with adjustments made for the chronic inflammatory state. Measurement of the soluble transferrin receptor might be a promising tool to accurately assess iron stores but may increase screening costs [39].

Patients with COPD are at increased risk for venous thromboembolism by virtue of their sedentary lifestyle, systemic inflammation and secondary polycythaemia associated with chronic hypoxia. According to a systematic review of literature by ALEVA et al. [40] in 2017, pulmonary embolism may have a prevalence of $16 \%$ in 
unexplained acute exacerbation of COPD, with increased mortality and length of hospitalisation.

Suspecting a diagnosis of pulmonary embolism often comes late, as the symptoms are nonspecific. However, correctly making the diagnosis is important, as even small clots may worsen COPD symptoms or have fatal consequences, due to the reduced cardiopulmonary reserve in COPD patients compared to patients without COPD. Modern generation $\mathrm{D}$-dimer testing, if negative, is useful to exclude venous thromboembolism. Computed tomography pulmonary angiography (CTPA) is reliable to diagnose pulmonary embolism if the study meets technical specifications, but is precluded in patients with significant renal impairment due to the risks of contrast nephropathy. Moreover, performing the study may not be possible in the setting of significant tachypnoea, due to the requirements for lying flat and breath-holding during the protocol. Ventilation/perfusion $\left(V^{\prime} / Q\right)$ scans are less reliable in COPD due to parenchymal lung disease and the resultant effects on the ventilation component of the study, complicating the assessment of $V^{\prime} / Q^{\prime}$ matching. Adding singlephoton emission computed tomography (SPECT) techniques may improve diagnostic sensitivity. Even with a technically suboptimal (nondiagnostic) study, it may be that the diagnosis can be confirmed by combining clinical suspicion, a $V^{\prime} / Q^{\prime}$ scan or computed tomography (CT) chest scan without contrast, and modalities such as venous ultrasound Doppler and echocardiogram.

\section{Lung cancer, sleep disturbance and sleep-related breathing disorders}

Screening for lung cancer and for the sleep disorders associated with COPD are both broad topics, beyond the scope of this review.

\section{Proposed comorbidity checklist during routine consultations for COPD}

During routine consultation in a stable COPD patient, the elements listed within table 2 should help to guide screening for comorbidities. Clinical examination should be as complete as possible. Taking a past medical history and reviewing the currently used medications are helpful to reveal which comorbidities have already been identified. It is of particular importance to ask specifically about symptoms of angina pectoris, depression, anxiety, swallowing problems, indigestion, musculoskeletal pain or limitation, sleep disorders, sinus or dental issues. This is also a good opportunity to encourage the patient to express their experience of life with COPD, the difficulties, what has been achieved, fears and expectations. The social and psychological impact of the disease are better appreciated by open discussion with the patient and family, and by sharing information between the various healthcare professionals who may be involved. It is always useful to take into account cultural differences, religious beliefs and language barriers. Physical examination should include measurement of $\mathrm{BMI}$, arterial blood pressure, pulse palpation and transcutaneous oxygen saturation, and then focus on the respiratory, cardiovascular and musculoskeletal systems. Any unusual symptom or physical sign should direct further attention and investigations. For example, haemoptysis, weight loss and/or clubbing may lead to lung cancer screening; orthopnoea, bilateral crackles and pedal oedema may prompt further investigations for heart failure.

Some complementary investigations may be helpful, as listed in table 2: a low-dose thorax

Table 2 Proposed comorbidity checklist during routine consultations for COPD

\begin{tabular}{llll}
\hline Past medical history & Symptoms & Physical examination & Screening tests \\
\hline High blood pressure & Angina pectoris & BMI & Low-dose thorax CT scan \\
Diabetes mellitus & Dyspnoea on exertion & Pulse palpation & Resting ECG \\
Dyslipidaemia & Orthopnoea & Arterial blood pressure & Echocardiography \\
Inactivity/exercise & Palpitations & $\mathrm{SpO}_{2}$ & CTCA and calcium score \\
Smoking & Claudication & Focus on respiratory, & 5-step approach for osteoporosis \\
GORD & Weight changes & cardiovascular and & Anxiety and depression scores (HADS, HSCL-25, \\
Falls & Musculoskeletal pain & musculoskeletal & CCQ, BDI) \\
Osteoporosis/fractures & Snoring, apnoea and & systems & EAT-10 or other screening tests for dysphagia \\
Psychological disorders & daytime sleepiness & & Blood tests: \\
Other respiratory & Sinus issues & & Haemoglobin, iron studies \\
disease & Dental issues & & BNP or NT-proBNP \\
Usual treatment & Swallowing disorders & & Fasting lipid profile \\
History of & Depression or anxiety & & Fasting blood glucose level, HbA1c \\
exacerbations & & & Vitamin D, serum calcium, phosphate \\
& & & Creatinine
\end{tabular}

$\mathrm{SpO}_{2}$ : oxygen saturation measured by pulse oximetry; NT-proBNP: N-terminal pro-BNP; HbA1c: glycated haemoglobin. 


\section{Self-evaluation questions}

1. Which of the following statements is/are true of COPD? Mark all that apply.
a. Defined only by airflow obstruction measured with spirometry
b. A multisystem disease
c. Often associated with comorbidities, which have an impact on prognosis
d. May be associated with chronic systemic inflammation

2. Which of the following statements is/are true of coronary artery disease? Mark all that apply.
a. Might be overlooked in COPD because of overlapping symptoms
b. Is often associated with COPD only because they share the same risk factor: tobacco smoking
c. Treatments of coronary artery disease are detrimental in COPD
d. Echocardiography is of no use in COPD because of
hypoechogenicity due to emphysema

3. Which of the following statements is/are true of atrial fibrillation? Mark all that apply.
a. Is very frequent during COPD exacerbation
b. May be asymptomatic or paroxysmal
c. Some treatments of COPD may induce or worsen arrhythmia
d. Can be detected by pulse palpation

4. Which of the following statements is/are true of metabolic diseases? Mark all that apply.
a. There is no link between diabetes and COPD
b. Dyslipidaemia is associated with better COPD outcomes
c. Obesity may be associated with malnutrition and sarcopenia
d. Metformin is contraindicated in stable COPD with diabetes

5. Which of the following statements is/are true of osteoporosis? Mark all that apply.
a. Osteoporosis associated with COPD is only due to systemic corticosteroid use
b. Vertebral fractures may be asymptomatic and have a negative effect on lung function
c. Low calcium level is a reliable diagnostic tool for osteoporosis
d. Supplementation with calcium and vitamin D may be proposed for treatment of osteoporosis in COPD

CT scan (as screening for lung cancer but which may also identify bronchiectasis or confirm the presence and extent of emphysema); a resting ECG, echocardiogram and CTCA with calcium score; and screening blood tests, including haemoglobin, natriuretic peptides (BNP or NT-proBNP), iron studies, fasting lipid profile and blood glucose level, HbA1c, vitamin D level, creatinine and electrolyte levels, according to local availability.

The checklist arises from the pooled experience of the authors, after years treating COPD patients in the outpatient setting. We hope that it might guide other clinicians in the management of COPD patients by incorporating assessment for the most frequently associated diseases and those most closely related to prognosis, leading to holistic, individualised care. Perhaps the next step would be to evaluate this tool in terms of its feasibility and utility in various real-world outpatient clinical settings, including specialist outpatient clinics and within primary care.

\section{Future directions}

Work is needed to better identify the most reliable and effective modalities to screen for comorbidities in COPD patients.

BNP is already known to be a sensitive marker of cardiac dysfunction. Looking ahead, given that BNP is a simple, inexpensive test, our recommendation is that it should be systematically measured in stable COPD patients, and an elevated level should prompt further investigations to confirm whether heart failure is present or to ascertain an alternative cause.

Atrial fibrillation is not currently screened extensively in the non-COPD population, even in those with established risk factors. COPD patients are at increased risk of both atrial fibrillation and cerebrovascular events. Hence, we propose that screening might be beneficial for COPD patients. A preliminary step to explore this might involve a prospective study comparing pulse palpation combined with ECG to 24-h and to 72-h ambulatory ECG in terms of reliability for detecting atrial fibrillation, with a view to determining the most reliable and cost-effective screening tool for atrial fibrillation in COPD.

Sarcopenia is another condition lacking a simple, easily accessible, low-cost tool for screening and, perhaps more importantly, for monitoring the effects of intervention. The development of a reliable, responsive clinical assessment tool for sarcopenia in individuals of obese or cachectic body morphology, using simple measurements that can be performed in any kind of health setting, is much needed.

With screening for lung cancer currently recommended in a subset of COPD patients, and CTCA evolving as perhaps the best screening test for high-risk coronary artery disease in COPD patients, we may need to work together with our radiology colleagues to develop a CT screening protocol to efficiently and reliably screen both conditions, obtaining the maximal amount of information at an acceptable level of radiation exposure.

In COPD patients identified as being at increased risk of coronary artery disease by COPDCoRi, or those with disproportionate dyspnoea on exertion, we propose that the calcium score should be evaluated routinely to seek evidence of atheromatous coronary involvement. A prospective study could be established, targeting stable COPD patients, comparing systematic screening for coronary artery disease by CTCA-derived calcium score (followed by treatment according to the results obtained) with standard care. The primary outcome sought would be the incident cardiac events occurring over a specified duration, with time to first coronary event as a secondary outcome. 
Research efforts should also clarify the potential effects of treating comorbidity on the evolution of COPD. Particularly, in the light of the recent favourable retrospective studies of metformin in COPD patients with diabetes [23, 24], prospective studies could be conducted to assess the value of instituting metformin therapy in COPD patients with type II diabetes, COPD patients with obesity (but no diabetes) and COPD controls with neither of these conditions, obviously with exclusions based on chronic gastrointestinal symptoms or being underweight. Another interesting research question to explore might be the role for metformin in COPD patients without diabetes but under treatment with short-course corticosteroids. Clearly, an issue of safety arises for metformin in the context of acute acidosis during COPD exacerbation, with concerns about adding to an existing respiratory acidosis the effects of lactic acidosis from high-dose $\beta$-agonist therapy and metformin treatment. Subsequent trials of metformin should monitor for this along with other safety outcomes.

\section{Conclusion}

The interaction between COPD and comorbidity provides a wide world to explore, perhaps leading to exciting new therapeutic implications, better relief of symptoms and improved quality of life in patients with COPD. The individual has regained the centre of attention, with opportunities to tailor the management plan according to individual characteristics and preferences. Many questions remain unanswered and afford motivation and opportunity for further clinical research.

\section{Suggested} answers

1. b, c, d.

2. a.

3. $a, b, c, d$.

4. b, c.

5. b, d.

\section{Key points}

- $\mathrm{COPD}$ is a multisystem disease, whose assessment should incorporate symptom severity, $\mathrm{FEV}_{1}$, history of exacerbations and comorbidities.

- While some comorbidities are easily recognisable because of their direct evident impact on COPD evolution or exacerbations, others are less obvious and need to be specifically sought in order to better manage COPD: cardiovascular diseases (coronary artery disease, arrhythmia), metabolic diseases (diabetes mellitus, dyslipidaemia, obesity, osteoporosis, sarcopenia), psychological disorders (anxiety, depression), lung cancer, sleep disruption and sleep disordered breathing, and GORD.

- Screening is relevant if a reliable diagnostic test and treatment are available, both at an acceptable cost/harm for the individual and for society.

- Active listening, clinical examination and teamwork are crucial elements of the evaluation of a person as a whole, and are unavoidable in COPD management.

- Identifying comorbid disease in the presence of COPD may be challenging, due to overlapping symptoms and the impact of COPD symptoms and pathophysiology on diagnostic tests used.

\section{Affiliations \\ Delphine Natali ${ }^{1}$, Georges Cloatre ${ }^{2}$, Philippe Hovette ${ }^{3}$, Belinda Cochrane ${ }^{4}$ \\ ${ }^{1}$ Respiratory Medicine Dept, Hanoi French Hospital, Hanoi, Vietnam. ${ }^{2}$ Cardiology Dept, Hanoi French Hospital, Hanoi, Vietnam. ${ }^{3}$ nternal Medicine Dept, Hanoi French Hospital, Hanoi, Vietnam. ${ }^{4}$ Dept of Respiratory and Sleep Medicine, Campbelltown Hospital, Sydney, Australia.}

\section{Conflict of interest}

D. Natali has nothing to disclose. G. Cloatre has nothing to disclose. P. Hovette has nothing to disclose. B. Cochrane has nothing to disclose.

\section{References}

1. Global Initiative for Chronic Obstructive Lung Disease (GOLD). Global Strategy for the Diagnosis, Management, and
Prevention of Chronic Obstructive Pulmonary Disease. 2019.

Available from: http://goldcopd.org/ 
2. Valderas JM, Starfield B, Sibbald B, et al. Defining comorbidity: implications for understanding health and health services Ann Fam Med 2009; 7: 357-363.

3. World Health Organization. Screening. www.who.int/cancer/ prevention/diagnosis-screening/screening/en/ Date last accessed: 27 April 2020.

4. Chatila WM, Thomashow BM, Minai OA, et al. Comorbidities in chronic obstructive pulmonary disease. Proc Am Thorac Soc 2008; 5: 549-555

5. Agusti A, Soriano JB. COPD as a systemic disease. COPD 2008; 5: 133-138.

6. Divo M, Cote C, de Torres JP, et al. Comorbidities and risk of mortality in patients with chronic obstructive pulmonary disease. Am J Respir Crit Care Med 2012; 186: 155-161.

7. Decramer M, Rennard S, Troosters T, et al. COPD as a lung disease with systemic consequences - clinical impact mechanisms, and potential for early intervention. COPD 2008; 5: 235-256.

8. Heindl S, Lehnert M, Criée CP, et al. Marked sympathetic activation in patients with chronic respiratory failure. $\mathrm{Am} J$ Respir Crit Care Med 2001; 164: 597-601.

9. Sakamaki F, Satoh T, Nagaya N, et al. Abnormality of left ventricular sympathetic nervous function assessed by 123/-metaiodobenzylguanidine imaging in patients with COPD Chest 1999; 116: 1575-1581

10. Hanlon P, Nicholl BI, Jani BD, et al. Examining patterns of multimorbidity, polypharmacy and risk of adverse drug reactions in chronic obstructive pulmonary disease: a crosssectional UK Biobank study. BMJ Open 2018; 8: e018404.

11. Hole DJ, Watt GC, Davey-Smith G, et al. Impaired lung function and mortality risk in men and women: findings from the Renfrew and Paisley prospective population study. BMJ 1996 313: 711-715.

12. Sin DD, Man SF. Chronic obstructive pulmonary disease as a risk factor for cardiovascular morbidity and mortality. Proc Am Thorac Soc 2005; 2: 8-11.

13. Salpeter S, Ormiston T, Salpeter E. Cardioselective betablockers for reversible airway disease. Cochrane Database Syst Rev 2002; 4: CD002992.

14. Salpeter S, Ormiston T, Salpeter E. Cardioselective betablockers for chronic obstructive pulmonary disease. Cochrane Database Syst Rev 2005; 4: CD003566.

15. Adabag AS, Wassif HS, Rice K, et al. Preoperative pulmonary function and mortality after cardiac surgery. Am Heart J 2010; 159: 691-697.

16. Konecny T, Somers K, Orban M, et al. Interactions between COPD and outcomes after percutaneous coronary intervention. Chest 2010; 138: 621-627.

17. Buch P, Friberg J, Scharling $\mathrm{H}$, et al. Reduced lung function and the risk of atrial fibrillation in the Copenhagen City Heart Study. Eur Respir J 2003; 21: 1012-1016.

18. García-Sanz MT, Cánive-Gómez JC, Senín-Rial L, et al. Oneyear and long-term mortality in patients hospitalized for chronic obstructive pulmonary disease. J Thorac Dis 2017 9: 636-645.

19. Steer J, Gibson J, Bourke SC. The DECAF Score: predicting hospital mortality in exacerbations of chronic obstructive pulmonary disease. Thorax 2012; 67: 970-976.

20. Cazzola M, Calzetta L, Matera MG et al. Chronic obstructive pulmonary disease and coronary disease: COPDCoRi, a simple and effective algorithm for predicting the risk of coronary artery disease in COPD patients. Respir Med 2015; 109 1019-1025.

21. Ozyilmaz S, Alisir MF, Serdar OA, et al. The value of coronary artery calcium score in the early diagnosis of coronary artery disease in patients with stable chronic obstructive pulmonary disease. Anatol J Cardiol 2016; 16: 283-289.
22. Lin CS, Liu CC, Yeh CC, et al. Diabetes risks and outcomes in chronic obstructive pulmonary disease patients: two nationwide population-based retrospective cohort studies. PLoS One 2017: 12: e0181815

23. Ho TW, Huang CT, Tsai YJ, et al. Metformin use mitigates the adverse prognostic effect of diabetes mellitus in chronic obstructive pulmonary disease. Respir Res 2019; 20: 69.

24. Yen FS, Chen W, Wei JC, et al. Effects of metformin use on total mortality in patients with type 2 diabetes and chronic obstructive pulmonary disease: a matched-subject design. PLoS One 2018; 13: e0204859.

25. Romme EA, Geusens P, Lems WF, et al. Fracture prevention in COPD patients; a clinical 5-step approach. Respir Res 2015; 16: 32.

26. Guo Y, Zhang T, Wang Z, et al. Body mass index and mortality in chronic obstructive pulmonary disease: a dose-response meta-analysis. Medicine 2016; 95: e4225.

27. Hoong JM, Ferguson M, Hukins C, et al. Economic and operational burden associated with malnutrition in chronic obstructive pulmonary disease. Clin Nutr 2017; 36: 1105-1109.

28. Ferreira IM, Brooks D, White J, et al. Nutritional supplementation for stable chronic obstructive pulmonary disease. Cochrane Database Syst Rev 2012; 12: CD000998.

29. Akner G, Larsson K. Undernutrition state in patients with chronic obstructive pulmonary disease. A critical appraisal on diagnostics and treatment. Respir Med 2016; 117: 81-91.

30. Gurgun A, Deniz S, Argin M, et al. Effects of nutritional supplementation combined with conventional pulmonary rehabilitation in muscle-wasted chronic obstructive pulmonary disease: a prospective, randomized and controlled study. Respirology 2013; 18: 495-500.

31. Cruz-Jentoft AJ, Baeyens JP, Bauer JM, et al. Sarcopenia: European consensus on definition and diagnosis. Report of the European Working Group on Sarcopenia in Older People. Age Ageing 2010; 39: 412-423.

32. Eisner MD, Blanc PD, Yelin EH, et al. Influence of anxiety on health outcomes in COPD. Thorax 2010; 65: 229-234.

33. Ng TP, Niti M, Tan WC, et al. Depressive symptoms and chronic obstructive pulmonary disease: effect on mortality, hospital readmission, symptom burden, functional status, and quality of life. Arch Intern Med 2007; 167: 60-67.

34. Bratås $\mathrm{O}$, Espnes GA, Rannestad $\mathrm{T}$, et al. Pulmonary rehabilitation reduces depression and enhances healthrelated quality of life in COPD patients - especially in patients with mild or moderate disease. Chron Respir Dis 2010; 7: 229-237

35. Martinez $\mathrm{CH}$, Okajima Y, Murray S, et al. Impact of self-reported gastroesophageal reflux in subjects from COPDGene cohort. Respir Res 2014; 15: 62

36. Regan J, Lawson S, De Aguiar V. The Eating Assessment Tool10 predicts aspiration in adults with stable chronic obstructive pulmonary disease. Dysphagia 2017; 32: 714-720.

37. Gonzalez Lindh M, Blom Johansson M, Jennische M, et al. Prevalence of swallowing dysfunction screened in Swedish cohort of COPD patients. Int J Chron Obstruct Pulmon Dis 2017; 12: 331-337.

38. Cvejic L, Harding R, Churchward T, et al. Laryngeal penetration and aspiration in individuals with stable COPD. Respirology 2011; 16: 269-275.

39. Beguin $Y$. Soluble transferrin receptor for the evaluation of erythropoiesis and iron status. Clin Chim Acta 2003; 329: 9-22.

40. Aleva FE, Voets LWLM, Simons SO, et al. Prevalence and localization of pulmonary embolism in unexplained acute exacerbations of COPD: a systematic review and metaanalysis. Chest 2017; 151: 544-554. 